\title{
The Metric Dimension of Two-Dimensional Extended Meshes
}

\author{
Ron $\operatorname{Adar}^{a}$ and Leah Epstein ${ }^{b}$
}

\begin{abstract}
We consider two-dimensional grids with diagonals, also called extended meshes or meshes. Such a graph consists of vertices of the form $(i, j)$ for $1 \leq i \leq m$ and $1 \leq j \leq n$, for given $m, n \geq 2$. Two vertices are defined to be adjacent if the $\ell_{\infty}$ distance between their vectors is equal to 1 . A landmark set is a subset of vertices $L \subseteq V$, such that for any distinct pair of vertices $u, v \in V$, there exists a vertex of $L$ with different distances to $u$ and $v$. We analyze the metric dimension and show how to obtain a landmark set of minimum cardinality.
\end{abstract}

Keywords: metric dimension, mesh graph, grid graph, landmark set, resolving set

\section{Introduction}

Consider an undirected graph $G=(V, E)$. For $u, v \in V$, let $d(u, v)$ denote the edge distance between these two vertices. A vertex $x \in V$ separates $u$ and $v$ if $d(x, u) \neq d(x, v)$, and in this case, $x$ is also called a separating vertex for $u$ and $v$. A landmark set (LS) is a subset $L \subseteq V$ such that for any pair of vertices $u \neq v, L$ has at least one vertex $y$ that separates $u$ and $v$. The vertices of a landmark set $L$ are often referred to as landmarks. In the algorithmic metric dimension problem, the goal is to find a landmark set $L$ of minimum cardinality. The cardinality of a minimum cardinality landmark set of $G$ is called the metric dimension of $G$. In a variant of the metric dimension problem (called the weighted metric dimension problem) the goal is to find a landmark set $L$ of minimum cost, where there is a non-negative cost function on $G$ 's vertices.

\footnotetext{
${ }^{a}$ Department of Computer Science, University of Haifa, Haifa, Israel. E-mail: radar03@csweb.haifa.ac.il

${ }^{b}$ Department of Mathematics, University of Haifa, Haifa, Israel. E-mail: lea@math.haifa.ac.il
} 
A two-dimensional extended mesh (or just mesh) $M_{m, n}$ (for integer parameters $m$ and $n$ ) has $|V|=m \cdot n$ vertices of the form $(i, j)$, where $1 \leq i \leq m$ and $1 \leq j \leq n$. For vertices $\left(i_{1}, j_{1}\right),\left(i_{2}, j_{2}\right)$, let $\left(\left(i_{1}, j_{1}\right),\left(i_{2}, j_{2}\right)\right) \in E$ if (and only if) $\max \left\{\left|i_{1}-i_{2}\right|,\left|j_{1}-j_{2}\right|\right\}=1$. The resulting distance between two vertices is the $\ell_{\infty}$ distance between their vectors, that is, $d\left(\left(i_{1}, j_{1}\right),\left(i_{2}, j_{2}\right)\right)=\max \left\{\left|i_{1}-i_{2}\right|,\left|j_{1}-j_{2}\right|\right\}$. This distance also called the chessboard distance. In accordance with the way we have defined the edges of the graph, it is the edge distance of $M_{m, n}$.

We number the rows of the mesh from top to bottom, and the columns from left to right. The rows are called $R_{1}, R_{2}, \ldots, R_{m}$ ( $R_{i}$ is also called row $i$ ), and the columns are called $C_{1}, C_{2}, \ldots, C_{m}\left(C_{j}\right.$ is also called column $\left.j\right)$. The $j$ th vertex of row $i$ is denoted by $(i, j)$.

A graph related to the two-dimensional mesh is the two-dimensional lattice, $G_{m, n}$, where there is an edge between vertices $\left(i_{1}, j_{1}\right),\left(i_{2}, j_{2}\right)$, if (and only if) $\left|i_{1}-i_{2}\right|+\left|j_{1}-j_{2}\right|=1$, and the resulting distance between two vertices is the $\ell_{1}$ distance between their vectors, that is, $d\left(\left(i_{1}, j_{1}\right),\left(i_{2}, j_{2}\right)\right)=\left|i_{1}-i_{2}\right|+\left|j_{1}-j_{2}\right|$. This distance also called the city block distance.

Former results involving mesh graph and lattice graph (both are also called grid graphs) can be found in $[15,14,16,13,2,1]$.

We survey some known results on mesh graphs. In the case $m=1$ and $n>1$ (or $n=1$ and $m>1$ ), $G_{m, n}$ is a path, and its metric dimension is known to be 1 [14]. In the case $m=n=1$, the graph has a single vertex, so the metric dimension is zero by definition. In [15], it was shown that for a two-dimensional lattice the metric dimension is always 2 (for $n \geq m \geq 2$ ), and for a two-dimensional mesh and $m=n \geq 2$, the metric dimension is 3 . In our previous work on two-dimensional lattice with a cost function on its vertices [1], we described a polynomial time algorithm for solving the weighted metric dimension problem.

In this work, we will extend the result in [15], and calculate the metric dimension of a two-dimensional mesh, for all values of $m, n \geq 2, m \neq n$. The metric dimension of $M_{m, n}$, denoted by $M D\left(M_{m, n}\right)$ is the minimum cardinality of any LS for $M_{m, n}$.

In this work our main goal is to prove the following theorem.

Theorem 1. The metric dimension of a mesh $M_{m, n}$ with $n>m \geq 2$ satisfies $M D\left(M_{m, n}\right)=\left\lceil\frac{n-1}{m-1}\right\rceil+1$.

We will prove a tight bound of $\left\lceil\frac{n-1}{m-1}\right\rceil+1$ on $M D\left(M_{m, n}\right)$. The lower bound is proved via a direct analysis of all possible column landmark sets (see formal definition on the next section), and for the upper bound we describe a specific landmark set of $M_{m, n}$, whose cardinality is $\left\lceil\frac{n-1}{m-1}\right\rceil+1$.

In the case where $n-1$ is divisible by $m-1$, the mesh contains $\frac{n-1}{m-1}$ concatenated square sub-meshes with $m$ rows and $m$ columns (where every two such sub-meshes 
share a single column). In this case we will show that each square sub-mesh (with $m$ rows and $m$ columns) has exactly two landmark vertices in a minimum cardinality LS. Note that substituting $m=n$ does not give the correct metric dimension for $m=n$ (which is 3 as stated above, and not 2), and this is a special case. Informally, the reason for this property is that once $M_{m, n}$ is not a square (i.e., $m \neq n$ ), given two vertices contained in a square sub-mesh of $M_{m, n}$, they can be separated by a vertex outside this sub-mesh, in some cases.

Interestingly, as a result of Theorem 1, one of the differences between a twodimensional lattice and a two-dimensional mesh, is that while the metric dimension of two-dimensional lattice is always 2, as quoted above, the metric dimension of two-dimensional mesh (for $n>m \geq 2$ ) grows as a function of $n$.

The first articles on the metric dimension problem were by Harary and Melter [11] and by Slater [18]. The problem is NP-hard [14] and hard to approximate [4, 9] for general graphs, and it was studied for specific graph classes $[11,18,14,6,3$, 17, 7, 5, 10]. Applications can be found in $[4,11,15,8,14,6]$, where some of these applications are relevant for weighted graphs (see also [10]).

\section{The column metric dimension and a lower bound}

In this section we define an auxiliary concept, which we use in order to provide a lower bound on $M D\left(M_{m, n}\right)$.

A Column Landmark Set (CLS) is a set $L \subseteq V$ such that for any pair of distinct vertices on one column, $\left(i_{1}, j\right)$ and $\left(i_{2}, j\right)$ (where $i_{1} \neq i_{2}$ ), there exists $u \in L$ such that $d\left(u,\left(i_{1}, j\right)\right) \neq d\left(u,\left(i_{2}, j\right)\right)$. The Column Metric Dimension of a graph $M_{m, n}$ is the minimum cardinality of any CLS for it, and it is denoted by $C M D\left(M_{m, n}\right)$.

Since any LS is a CLS (as all vertices are separated by some vertex of LS, this clearly holds for pairs of vertices on the same column), we have for any mesh $G$ that $M D\left(M_{m, n}\right) \geq C M D\left(M_{m, n}\right)$. In this section we would like to prove a lower bound on $M D\left(M_{m, n}\right)$, and for that we will prove the following lemma.

Lemma 1. The column metric dimension of a mesh $M_{m, n}$ with $m, n \geq 2$ satisfies $C M D\left(M_{m, n}\right) \geq\left\lceil\frac{n-1}{m-1}\right\rceil+1$. Thus, in the case $n>m$, we have $M D\left(M_{m, n}\right) \geq$ $\left\lceil\frac{n-1}{m-1}\right\rceil+1$.

Note that the lower bound on $C M D\left(M_{m, n}\right)$ is proved for any $m, n \geq 2$ and not only for the case $n>m$. In what follows we consider these more general cases. Before we prove the lemma, we state and prove several simple and useful claims. Consider specific values $m, n \geq 2$, and a specific CLS $L$ for this graph. Let $\mu_{j}$ be the number of elements of $L$ in column $C_{j}$ (that is $\mu_{j}=\left|C_{j} \cap L\right|$ ), 
and let $N_{j}=\sum_{\ell=1}^{j} \mu_{j}$ be the number of landmarks in the first $j$ columns (i.e., $\left.N_{j}=\left|\left(C_{1} \cup C_{2} \cup \cdots \cup C_{j}\right) \cap L\right|\right)$. We let $N_{j}=N_{n}$ for $j>n$.

The goal of the first claim is to provide a lower bound on the number of landmarks in the first few columns. We will use this claim for the last $j$ columns as well (this follows from symmetry).

Claim 1. Consider vertices $u_{1}=\left(x_{1}, y^{\prime}\right)$ and $u_{2}=\left(x_{2}, y^{\prime}\right)$ (on column $\left.y^{\prime}\right)$. Let $v=(x, y)$ be a vertex that separates them, that is $d\left(v, u_{1}\right) \neq d\left(v, u_{2}\right)$. Then, $\left|y-y^{\prime}\right| \leq m-2$.

Proof. Assume by contradiction that $\left|y-y^{\prime}\right| \geq m-1$. Then, for $k=1,2, d\left(v, u_{k}\right)=$ $\max \left\{\left|x-x_{k}\right|,\left|y-y^{\prime}\right|\right\}=\left|y-y^{\prime}\right|$, as $\left|x-x_{k}\right| \leq m-1$ while $\left|y-y^{\prime}\right| \geq m-1$, so the distances to $v$ are determined by the columns. This contradicts $d\left(v, u_{1}\right) \neq$ $d\left(v, u_{2}\right)$.

Claim 2. The inequality $N_{\left\lceil\frac{m}{2}\right\rceil} \geq 1$ holds for any CLS L. Moreover, if $N_{1}=0$, then $N_{m-1} \geq 2$.

Proof. If $N_{1}>0$, the first part holds, so we assume $N_{1}=0$ and prove both parts of the claim. Consider two vertices on column $1, a_{1}=\left(\left\lfloor\frac{m}{2}\right\rfloor, 1\right)$ and $a_{2}=\left(\left\lfloor\frac{m}{2}\right\rfloor+1,1\right)$ (for even values of $m$ they are exactly in the middle of column 1 , for odd values of $m$ they are approximately in the middle of column 1 ). Note that $\left\lfloor\frac{m}{2}\right\rfloor \geq 1$ and $\left\lfloor\frac{m}{2}\right\rfloor+1 \leq m$, for $m \geq 2$, so these two vertices are well defined.

Let $b=\left(x_{b}, y_{b}\right)$ denote a vertex of $L$ separating $a_{1}$ and $a_{2}$. We will show $y_{b} \leq$ $\left\lceil\frac{m}{2}\right\rceil$, proving $N_{\left\lceil\frac{m}{2}\right\rceil} \geq 1$. Assume by contradiction $y_{b} \geq\left\lceil\frac{m}{2}\right\rceil+1$. We will show that the distance of $b$ to $a_{1}$ and $a_{2}$ is defined by the difference between column indices and therefore the distances are equal. We get $d\left(a_{1}, b\right)=\max \left\{\left|x_{b}-\left\lfloor\frac{m}{2}\right\rfloor\right|,\left|y_{b}-1\right|\right\}$ and $d\left(a_{2}, b\right)=\max \left\{\left|x_{b}-\left(\left\lfloor\frac{m}{2}\right\rfloor+1\right)\right|,\left|y_{b}-1\right|\right\}$. Since $1 \leq x_{b} \leq m$, we get $\left|x_{b}-\left\lfloor\frac{m}{2}\right\rfloor\right| \leq$ $\left\lceil\frac{m}{2}\right\rceil \leq y_{b}-1$ and $\left|x_{b}-\left\lfloor\frac{m}{2}\right\rfloor-1\right| \leq\left\lceil\frac{m}{2}\right\rceil \leq y_{b}-1$, so $d\left(a_{1}, b\right)=d\left(a_{2}, b\right)=y_{b}-1$, a contradiction.

Next, we analyze $N_{m-1}$ for the case $N_{1}=0$. Since $N_{m-1}>0$ (as $m-1 \geq\left\lfloor\frac{m}{2}\right\rfloor$ for $m \geq 2$ ), there is at least one vertex $c \in L$ in this case, as we have shown. It remains to show that there is a pair of vertices in the first $m-1$ columns not separated by $c$, implying $|L| \geq 2$. Let $c=\left(x_{c}, y_{c}\right)$ be a vertex of $L$ such that $2 \leq y_{c} \leq m-1$. Once again we consider two vertices of the first column, and show that $c$ does not separate them. Let $a_{1}^{\prime}=\left(x_{c}, 1\right)$, i.e., the vertex of the first column on the same row as $c$. If $x_{c}=1$, let $a_{2}^{\prime}=\left(x_{c}+1,1\right)$ and otherwise $a_{2}^{\prime}=\left(x_{c}-1,1\right)$. Thus, $a_{2}^{\prime}$ is well defined, as it is either the vertex just above $a_{1}^{\prime}$ or just below it (since $m \geq 2$, at least one of these vertices exists). We have $d\left(a_{1}^{\prime}, c\right)=\max \left\{0,\left|y_{c}-1\right|\right\}=y_{c}-1$ and $d\left(a_{2}^{\prime}, c\right)=\max \left\{1,\left|y_{c}-1\right|\right\}=y_{c}-1$, since $y_{c} \geq 2$. Thus, as $L$ contains a vertex separating $a_{1}^{\prime}$ and $a_{2}^{\prime}$, and by Claim 1 this 
vertex is on one of the first $m-1$ columns (or of the $n$ columns, if $n<m-1$ ), we get $N_{m-1} \geq 2$.

Claim 3. Let $L$ be a $C L S$ defined for $M_{m, n}$ such that $m, n \geq 2$ (where each element of $L$ is of the form $(i, j)$ ). If $L \cap C_{n}=\emptyset$, then $L$ is a $C L S$ for $M_{m, n-1}$. If $L \cap R_{m}=\emptyset$, then $L$ is a $C L S$ for $M_{m-1, n}$.

Proof. Consider two vertices $\left(i_{1}, j_{1}\right)$ and $\left(i_{2}, j_{2}\right)$. Any shortest path between these two vertices traverses only vertices on columns $\min \left\{j_{1}, j_{2}\right\}, \ldots, \max \left\{j_{1}, j_{2}\right\}$ and rows $\min \left\{i_{1}, i_{2}\right\}, \ldots, \max \left\{i_{1}, i_{2}\right\}$, that is, on columns and rows between the columns of these vertices and rows between the rows of these vertices. This implies the validity of the claim.

Proof. We now prove Lemma 1. We start this proof with several simple cases, which will allow us to use induction for the remaining cases $($ on $n+m)$.

Case 1. Consider the case $m=2$. In this case we show $C M D\left(M_{m, n}\right) \geq n$. To prove this, we show that $\mu_{j} \geq 1$ for $1 \leq j \leq n$. By Claim 1 , the only vertices that separate the two vertices $(1, j)$ and $(2, j)$ are on column $j$, that is, one of these two vertices. Therefore, any CLS either contains at least one of $(1, j)$ and $(2, j)$.

Case 2. Consider the case $n \leq m$ (where $m \geq 3$ ). In this case we show $\left.C M D\left(M_{m, n}\right)\right) \geq 2$. If $\mu_{1} \geq 1$ and $\mu_{n} \geq 1$, we are done. Otherwise, at least one of the columns $C_{1}$ and $C_{n}$ does not have a landmark. Assume without loss of generality (by rotating the mesh by 180 degrees or by reflecting it across a vertical line) that $L \cap C_{1}=\emptyset$. By Claim $2, N_{m-1} \geq 2$, so $|L| \geq 2$.

We are left with the case $n>m \geq 3$. We say that a gap (with respect to a CLS $L$ ) is a sequence of $m-1$ columns that do not contain elements of $L$, that is, there is an index $1 \leq k \leq n-m+2$ such that $\left\{C_{k}, C_{k+1}, \ldots, C_{k+m-2}\right\} \cap L=\emptyset$. The cases $k=1$ and $k=n-m+2$ are not possible due to Claim 2, as the first $m-1$ columns have at least one element of $L$, and symmetrically, the last $m-1$ columns have at least one element of $L$. Thus, $k$ satisfies $2 \leq k \leq n-m+1$, and in particular, a gap is possible only if $n \geq m+1$ (since all cases where $n \leq m$ were already considered, a gap is defined for all remaining cases).

We use the first two cases as the induction base, and prove the remaining cases via induction. 
Case 3. There is a gap in $L$. Let $k(2 \leq k \leq n-m+1)$ be such that $\left\{C_{k}, C_{k+1}, \ldots, C_{k+m-2}\right\} \cap L=\emptyset$, that is, all elements of $L$ are on columns $C_{1}, C_{2} \ldots$, $C_{k-1}, C_{k+m-1}, \ldots, C_{n}$. Let $L_{1}=\left\{C_{1}, C_{2}, \ldots, C_{k}\right\} \cap L$ and let $L_{2}=\left\{C_{k+m-2}\right.$, $\left.C_{k+m-1}, \ldots, C_{n}\right\} \cap L$ (so $L=L_{1} \cup L_{2}$ and $L_{1} \cap L_{2}=\emptyset$ since $m \geq 3$ ). We claim that $L_{1}$ is a CLS for the sub-mesh of $k$ columns and $m$ rows consisting of the first $k \geq 2$ columns. Since $L$ is a CLS, for every pair of distinct vertices $v_{1}$ and $v_{2}$ on one of the first $k$ columns, there is a vertex of $L$ separating them. By Claim 1 , such a vertex is not on columns $k+m-1, \ldots, n$. As columns $k, k+1, \ldots, k+m-2$ have no elements of $L$, there is an element of $L$ separating $v_{1}$ and $v_{2}$ on one of the columns $1,2, \ldots, k-1$, that is, it is an element of $L_{1}$. Thus, $L_{1}$ is a CLS for a mesh of $k$ columns and $m$ rows. Analogously, it is possible to prove that $L_{2}$ is a CLS for a mesh of $n-m+3-k \geq 2$ columns and $m$ rows. Using induction, and as the numbers of columns of $L_{1}$ and $L_{2}$ are $k$ and $n-m-k+3$, respectively (with $m$ rows), we find $|L|=\left|L_{1}\right|+\left|L_{2}\right| \geq\left\lceil\frac{k-1}{m-1}\right\rceil+1+\left\lceil\frac{(n-m+3-k)-1}{m-1}\right\rceil+1 \geq 2+\left\lceil\frac{n-m+1}{m-1}\right\rceil=$ $\left\lceil\frac{n}{m-1}\right\rceil+1 \geq\left\lceil\frac{n-1}{m-1}\right\rceil+1$, which holds by properties of rounding up.

We are left with the case where there is no gap in $L$. For an integer $c \geq 0$, let $h_{c}=m+(m-1) c$ and $h_{c}^{\prime}=m-1+(m-1) c=h_{c}-1$. Note that by their definitions it holds that $m \leq h_{c}$ and $m-1 \leq h_{c}^{\prime}$ for any value of $c$. We claim that in this case, if $h_{c} \leq n$, we have $N_{h_{c}} \geq c+2$, and additionally, if $h_{c}^{\prime} \leq n$ and $N_{1}=0$ hold, $N_{h_{c}^{\prime}} \geq c+2$. This is proved by induction on $c$. For $c=0$, if $N_{1}=0$, by Claim 2, $N_{m} \geq N_{m-1} \geq 2$. Otherwise, $N_{1} \geq 1$, and since there are no gaps, at least one of the columns $C_{2}, C_{3}, \ldots, C_{m}$ has an element of $L$, so $N_{m} \geq 2$. The inductive step is proved in a similar manner. If $N_{h_{c-1}} \geq c+1$ for some integer $c \geq 1$, as there is no gap, there is at least one element of $L$ on columns $h_{c-1}+1, h_{c-1}+2, \ldots, h_{c-1}+m-1$. Since $h_{c-1}=m+(m-1)(c-1)=1+(m-1) c$ we get that $h_{c-1}+m-1=h_{c}$, and as a result $N_{h_{c}} \geq N_{h_{c-1}}+1 \geq c+2$. If $N_{h_{c-1}^{\prime}} \geq c+1$ for some $c \geq 1$, as there is no gap, there is at least one element of $L$ on columns $h_{c-1}^{\prime}+1, h_{c-1}^{\prime}+2, \ldots, h_{c-1}^{\prime}+m-1$. Since $h_{c-1}^{\prime}=h_{c-1}-1=(m-1) c$ we get that $h_{c-1}^{\prime}+m-1=h_{c-1}-1=h_{c}^{\prime}$, and as a result $N_{h_{c}^{\prime}} \geq N_{h_{c-1}^{\prime}}+1 \geq c+2$.

Case 4. There is an element of $L$ on the first column or on the last column (or both). Without loss of generality (by possibly rotating the mesh by 180 degrees) we assume $L \cap C_{n} \neq \emptyset$, i.e., $\mu_{n}>0$. Let $c=\left\lceil\frac{n-1}{m-1}\right\rceil-2$, where $c \geq 0$ as $n>m$. Consider columns $1,2, \ldots, m+(m-1) c$, where $(m-1) c+m<(m-1) \cdot\left(\frac{n-1}{m-1}-1\right)+m=n$. Thus, as $h_{c} \leq n-1$, we have $|L| \geq N_{h_{c}}+\mu_{n} \geq c+3=\left\lceil\frac{n-1}{m-1}\right\rceil+1$.

We are left with the case where $\mu_{1}=0$ and $\mu_{n}=0$.

Case 5. The number of columns is sufficiently large, that is, $n \geq 2 m$. Let $c=$ $\left\lfloor\frac{n}{m-1}\right\rfloor-2$, where $c \geq 0$ as $n>m$. Consider columns $1,2, \ldots, m-1+(m-1) c$, 
where $m-1+(m-1) c=(m-1)(c+1) \leq(m-1) \cdot\left(\frac{n}{m-1}-1\right)=n-m+1$. By Claim 2, the last $m-1$ columns have at least two elements of $L$. Thus, $|L| \geq$ $N_{h_{c}^{\prime}}+2 \geq c+4=\left\lfloor\frac{n}{m-1}\right\rfloor+2 \geq\left\lceil\frac{n}{m-1}\right\rceil+1 \geq\left\lceil\frac{n-1}{m-1}\right\rceil+1$.

We are left with the case where $n$ satisfies $3 \leq n \leq 2 m-1$.

Case 6. The number of columns satisfies $m+2 \leq n \leq 2 m-1$. Since $C_{n}$ has no elements of $L$, using Claim 3, $L$ is a CLS for the sub-mesh consisting of first $n-1 \geq m+1$ columns and $m$ rows, and by induction, $|L| \geq 3$.

Case 7. The number of columns satisfies $n=m+1$, and at least one row out of $R_{1}$ and $R_{m}$ has no element of $L$ (i.e., at least one of the following holds: $R_{1} \cap L=\emptyset$, $R_{m} \cap L=\emptyset$ ). In this case, using Claim $3, L$ is a CLS for a mesh of $m-1$ rows and $n$ columns. Since $n=(m-1)+2$, using induction we have $|L| \geq 3$.

We are left with the case where $n=m+1,\left|L \cap R_{1}\right| \geq 1$ and $\left|L \cap R_{m}\right| \geq 1$. Notice that the case where $|L| \geq 3$ satisfies the demand since $|L| \geq\left\lceil\frac{m}{m-1}\right\rceil+1=3$.

The last case to consider is $n=m+1,|L| \leq 2$, so $\left|L \cap R_{1}\right|=1$ and $\left|L \cap R_{m}\right|=1$, and no other row has an element of $L$. We show that this scenario is not possible.

Case 8. Let $L=\left\{\left(1, y_{1}\right),\left(m, y_{2}\right)\right\}$. Since $C_{1}$ and $C_{n}$ have no elements of $L$, we have $2 \leq y_{1}, y_{2} \leq n-1$. By Claim $2, L$ has at least one element on columns $C_{1}, C_{2}, \ldots, C_{\left\lceil\frac{m}{2}\right\rceil}$ and symmetrically at least one element on columns $C_{\left\lfloor\frac{m}{2}\right\rfloor+2}, \ldots$, $C_{m+1}$. As $\left\lceil\frac{m}{2}\right\rceil<\left\lfloor\frac{m}{2}\right\rfloor+2$, we find that one of $y_{1}, y_{2}$ is in $\left\{1,2, \ldots,\left\lceil\frac{m}{2}\right\rceil\right\}$, and the other is in $\left\{\left\lfloor\frac{m}{2}\right\rfloor+2, \ldots, m+1\right\}$. Without loss of generality, by possibly reflecting the mesh across a vertical line, assume that $y_{1} \leq\left\lceil\frac{m}{2}\right\rceil$ and $y_{2} \geq\left\lfloor\frac{m}{2}\right\rfloor+2$. Moreover, by possibly rotating the mesh by 180 degrees, we assume that $y_{1}$ is at least as far from the first column as $y_{2}$ is to the last column, that is, $y_{1}-1 \geq n-y_{2}=m+1-y_{2}$. Consider the vertices $v_{1}=\left(y_{1}-1,1\right)$ and $v_{2}=\left(y_{1}, 1\right)$. Note that $v_{1}$ is well defined since $y_{1} \geq 2$. If $L$ is a CLS, these two vertices are separated by $\left(1, y_{1}\right)$ or by $\left(m, y_{2}\right)$. We have $\left.d\left(\left(1, y_{1}\right), v_{1}\right)=d\left(1, y_{1}\right), v_{2}\right)=y_{1}-1$, so $\left(1, y_{1}\right)$ does not separate $v_{1}$ and $v_{2}$. We also have $d\left(\left(m, y_{2}\right), v_{1}\right)=\max \left\{m-y_{1}+1, y_{2}-1\right\}$ and $d\left(\left(m, y_{2}\right), v_{1}\right)=\max \left\{m-y_{1}, y_{2}-1\right\}$. Since $y_{2}-1 \geq m-y_{1}+1>m-y_{1}$, we get $d\left(\left(m, y_{2}\right), v_{1}\right)=d\left(\left(m, y_{2}\right), v_{2}\right)=y_{2}-1$, so $v_{1}$ and $v_{2}$ are not separated by a vertex of $L$, a contradiction.

\section{An upper bound}

Given $M_{m, n}$ with $n \geq m \geq 2$, we define a set $L \subseteq V$ and show that its cardinality is according to Theorem 1 (i.e., $\left\lceil\frac{n-1}{m-1}\right\rceil+1$ ), and that it is a landmark set (from now 
on we only consider LS not CLS like in the previous section). Let $f=\left\lfloor\frac{n-1}{m-1}\right\rfloor+1$. For $1 \leq k \leq f$, if $k$ is odd, let $z_{k}=(1,1+(k-1)(m-1))$, and if $k$ is even, let $z_{k}=(m, 1+(k-1)(m-1))$. Note that $1+(f-1)(m-1) \leq 1+\frac{n-1}{m-1} \cdot(m-1)=n$, so $z_{1}, z_{2}, \ldots, z_{f}$ are well-defined. That is, there is an element of $L$ every $m-1$ columns, starting with the first column. The rows of these elements alternate between 1 and $m$. If $1+(f-1)(m-1)=n$, that is, $n-1$ is divisible by $m-1$, vertex $z_{f}$ is on column $n$, moreover, in this case, $\left\lceil\frac{n-1}{m-1}\right\rceil=\left\lfloor\frac{n-1}{m-1}\right\rfloor$, and we have defined $L$ completely. If $1+(f-1)(m-1)<n$, that is, $n-1$ is not divisible by $m-1$, we have $\left\lceil\frac{n-1}{m-1}\right\rceil=\left\lfloor\frac{n-1}{m-1}\right\rfloor+1$, and we define $z_{f+1}=(1, n)$ if $f$ is even (and $f+1$ is odd), and otherwise we let $z_{f+1}=(m, n)$.

Let $f^{\prime}=\left\lceil\frac{n-1}{m-1}\right\rceil+1$. The elements of $L$ are $z_{1}, \ldots, z_{f^{\prime}}$ (no matter whether $f^{\prime}>f$ or $\left.f^{\prime}=f\right)$. Figure 1 shows an example of the set $L$ for a mesh with $m=5$ and $n=12$.

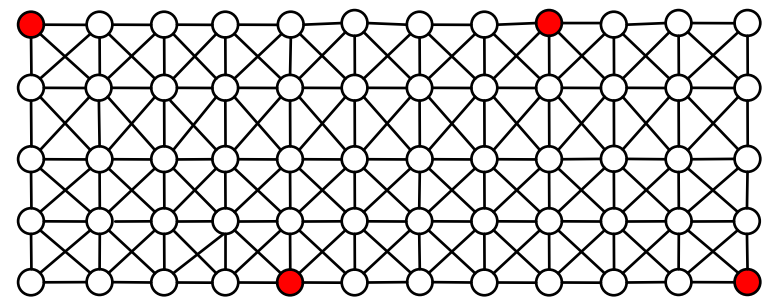

$(5,5)$

Figure 1: An example of the set $L=\{(1,1),(5,5),(1,9),(5,12)\}$ described in the above explanation, for a mesh with 5 rows and 12 columns.

Claim 4. Consider the case $n \geq 2 m-1$, and consider two vertices $v_{1}=\left(x_{1}, y_{1}\right)$ and $v_{2}=\left(x_{2}, y_{2}\right)$. If neither $z_{1}$ nor $z_{f^{\prime}}$ separates $v_{1}$ and $v_{2}$, then $v_{1}$ and $v_{2}$ are on the same column (that is, $y_{1}=y_{2}$ ).

Proof. Assume by contradiction that $y_{1} \neq y_{2}$ and assume without loss of generality that $y_{1}<y_{2}$. If $y_{2} \geq m+1$, we have $d\left(v_{2}, z_{1}\right)=\max \left\{x_{2}-1, y_{2}-1\right\}$. Since $x_{2}-1 \leq m-1$ while $y_{2}-1 \geq m$, we have $d\left(v_{1}, z_{1}\right)=d\left(v_{2}, z_{1}\right)=y_{2}-1$ where the first equality holds as $z_{1}$ does not separate $v_{1}$ and $v_{2}$. Next, by $y_{2}-1=d\left(v_{1}, z_{1}\right)$ and $d\left(v_{1}, z_{1}\right)=\max \left\{x_{1}-1, y_{1}-1\right\}$, and since $x_{1}-1 \leq m-1$, we get $d\left(v_{1}, z_{1}\right)=y_{1}-1$. Thus $y_{1}=y_{2}$ in this case, a contradiction.

If $y_{2} \leq m$, we get $y_{1} \leq m-1$, and the proof is symmetric for the distances from $z_{2}$ instead of $z_{1}$ (by rotating the mesh and possibly reflecting it across a horizontal 
line); the distance of $v_{1}$ to $z_{2}$ is at least $m$, and since $v_{2}$ has the same distance, the maximum value of the distance is achieved by the second term also for $v_{2}$ and they are on one column.

Claim 5. Consider two vertices $u_{1}=(1,1)$ and $u_{2}=(m, q)$, such that $2 \leq q \leq n$ and $q \leq m$. Consider also two distinct vertices $v_{1}=\left(x_{1}, y\right)$ and $v_{2}=\left(x_{2}, y\right)$ (on one column), where $1 \leq x_{1}<x_{2} \leq m$ and $1 \leq y \leq q$. Then, at least one of $u_{1}$ and $u_{2}$ separates $v_{1}$ and $v_{2}$.

Proof. If $x_{1} \geq y$, we have $x_{2} \geq y+1$. Thus, for $i=1,2, d\left(v_{i}, u_{1}\right)=\max \left\{x_{i}-\right.$ $1, y-1\}=x_{i}-1$, and since $x_{1}-1 \neq x_{2}-1$, we find that $u_{1}$ separates $v_{1}$ and $v_{2}$. Otherwise, $x_{1}<y$ holds, and we show that $u_{2}$ separates $v_{1}$ and $v_{2}$. So $d\left(v_{1}, u_{2}\right)=$ $\max \left\{m-x_{1}, q-y\right\}$, and since $q \leq m,-y<-x_{1}$, we find $d\left(v_{1}, u_{2}\right)=m-x_{1}$. On the other hand, $d\left(v_{2}, u_{2}\right)=\max \left\{m-x_{2}, q-y\right\}$, where $m-x_{2}<m-x_{1}$ (as $\left.x_{1}<x_{2}\right)$ and $q-y<m-x_{1}$, so $d\left(v_{2}, u_{2}\right)=\max \left\{m-x_{2}, q-y\right\}<d\left(v_{1}, u_{2}\right)$, and therefore $u_{2}$ separates $v_{1}$ and $v_{2}$.

Corollary 1. Consider two vertices $u_{1}=(1,1)$ and $u_{1}=(m, q)$, such that $2 \leq$ $q \leq n$ and $q \geq m$. Consider two distinct vertices $v_{1}=\left(x, y_{2}\right)$ and $v_{2}=\left(x, y_{2}\right)$ (on one row), where $1 \leq y_{1}<y_{2} \leq q$ and $1 \leq x \leq m$. Then, at least one of $u_{1}$ and $u_{2}$ separates $v_{1}$ and $v_{2}$.

The corollary holds by rotating the mesh by 90 degrees.

Claim 6. Consider two distinct vertices $v_{1}=\left(x_{1}, y\right)$ and $v_{2}=\left(x_{2}, y\right)$ (on one column $)$. Let $z_{s}=\left(x_{s}, y_{s}\right)$ and $z_{s+1}=\left(x_{s+1}, y_{s+1}\right)$ be such that $y_{s} \leq y \leq y_{s+1}$. Then, at least one of $z_{s}$ and $z_{s+1}$ separates $v_{1}$ and $v_{2}$.

Proof. Without loss of generality it is sufficient to consider the sub-graph of the mesh consisting of columns $y_{s}, y_{s}+1, \ldots, y_{s+1}$ and all rows. The property holds by Claim 5, as no shortest path between two vertices in this sub-mesh traverses any vertex outside it, and by possibly reflecting the mesh across a vertical line.

Corollary 2. In the case $n \geq 2 m-1$, the set $L$ is a landmark set.

Proof. By Claim 4, for every pair of vertices on different columns, $L$ contains a vertex separating them. By Claim 6, every pair of vertices on one column is separated as well, by choosing an appropriate value of $s$, which is possible for any $y$ since $L$ has an element on $C_{1}$ and an element on $C_{n}$.

We are left with the case where $n$ satisfies $m+1 \leq n \leq 2 m-2$ (in particular, $n \geq 3)$. In this case, $L=\left\{z_{1}=(1,1), z_{2}=(m, m), z_{3}=(1, n)\right\}$. 
Claim 7. Consider two distinct vertices $v_{1}=\left(x_{1}, y_{1}\right)$ and $v_{2}=\left(x_{2}, y_{2}\right)$, in the case where $n \in\{m+1, m+2, \ldots, 2 m-2\}$. At least one of the vertices $z_{1}$ and $z_{3}$ separates $v_{1}$ and $v_{2}$.

Proof. Assume that $z_{1}$ does not separate $v_{1}$ and $v_{2}$. We have $d\left(v_{i}, z_{1}\right)=\max \left\{x_{i}-\right.$ $\left.1, y_{i}-1\right\}$.

If $x_{1}=x_{2}$, we have $y_{1} \neq y_{2}$. By $\max \left\{x_{1}-1, y_{1}-1\right\}=\max \left\{x_{2}-1, y_{2}-1\right\}$ we find that it cannot be the case that $d\left(v_{i}, z_{1}\right)=y_{i}-1$ for $i=1,2$. Thus, we can assume without loss of generality that $d\left(v_{1}, z_{1}\right)=x_{1}-1>y_{1}-1$. We claim that $x_{2} \geq y_{2}$ holds. Indeed, $d\left(v_{2}, z_{1}\right)=d\left(v_{1}, z_{1}\right)=x_{1}-1=x_{2}-1$, so $x_{2}-1 \geq y_{2}-1$. Thus, as $x_{1}=x_{2} \leq m$, we have $y_{1}<m$ and $y_{2} \leq m$, and by Corollary $1, z_{2}=(m, m)$ separates $v_{1}$ and $v_{2}$.

If $y_{1}=y_{2}$, by Claim 6 , one of $z_{1}, z_{2}$, and $z_{3}$ separates $v_{1}$ and $v_{2}$.

We are left with the case $x_{1} \neq x_{2}$ and $y_{1} \neq y_{2}$. By the definition of distances, we cannot have that $d\left(v_{i}, z_{1}\right)=x_{i}-1$ holds for $i=1,2$ or that $d\left(v_{i}, z_{1}\right)=y_{i}-1$ holds for $i=1,2$, so we have either $d\left(v_{1}, z_{1}\right)=y_{1}-1$ and $d\left(v_{2}, z_{1}\right)=x_{2}-1$ (and $\left.x_{1}<y_{1}, x_{2}>y_{2}\right)$ or we have $d\left(v_{1}, z_{1}\right)=x_{1}-1$ and $d\left(v_{2}, z_{1}\right)=y_{2}-1$ (and $x_{1}>y_{1}$, $x_{2}<y_{2}$ ). Without loss of generality (by possibly swapping the roles of $v_{1}$ and $v_{2}$ ) we assume that $d\left(v_{1}, z_{1}\right)=y_{1}-1$ and $d\left(v_{2}, z_{1}\right)=x_{2}-1$ holds, so $y_{1}=x_{2}$, and $x_{1}<y_{1}, x_{2}>y_{2}$.

Consider the distances to $z_{3}$. We find $d\left(v_{1}, z_{3}\right)=\max \left\{x_{1}-1, n-y_{1}\right\}$ and $d\left(v_{2}, z_{3}\right)=\max \left\{x_{2}-1, n-y_{2}\right\}$. Assume that $d\left(v_{1}, z_{3}\right)=x_{1}-1$ holds. If $d\left(v_{2}, z_{3}\right)=$ $x_{2}-1$, we are done by $x_{1} \neq x_{2}$. Otherwise $d\left(v_{2}, z_{3}\right)=n-y_{2}$, and we have $x_{1}-1>n-y_{1}$ and $x_{2}-1<n-y_{2}$. We get $n-y_{2}>x_{2}-1=y_{1}-1>x_{1}-1$. Assume that $d\left(v_{1}, z_{3}\right)=n-y_{1}$ holds. If $d\left(v_{2}, z_{3}\right)=n-y_{2}$, we are done by $y_{1} \neq y_{2}$. Otherwise $d\left(v_{2}, z_{3}\right)=x_{2}-1$, and we have $x_{1}-1<n-y_{1}$ and $x_{2}-1>n-y_{2}$. We get $x_{2}-1>n-y_{2}>n-x_{2}=n-y_{1}$, so $z_{3}$ separates $v_{1}$ and $v_{2}$ in all remaining cases.

\section{Conclusion}

In section 2 we have proved a lower bound of $\left\lceil\frac{n-1}{m-1}\right\rceil+1$ on the column metric dimension of $M_{m, n}$, for all values of $m, n \geq 2$, implying the lower bound for the metric dimension for $n>m \geq 2$. In section 3 we have proved an upper bound of $\left\lceil\frac{n-1}{m-1}\right\rceil+1$ on the metric dimension of $M_{m, n}$, for all values of $n>m \geq 2$, by defining a suitable landmark set. As mentioned in Section 1, the case of $m=n$ is a special case where $M D\left(M_{m, n}\right)=3$ [15], which was known prior to our work, and we do not analyze this case. Our main result (Theorem 1) is proved by combining the lower bound and upper bound. Using the last remark we get a full characterization of the metric dimension of (two-dimensional) meshes. 


\section{References}

[1] R. Adar, L. Epstein. An algorithm for the weighted metric dimension of twodimensional grids. arXiv:1602.05899

[2] P. Andersen, C. Grigorious, and M. Miller. Minimum weight resolving sets of grid graphs. Discrete Mathematics, Algorithms and Applications, 8(3), 22 pages.

[3] L. Babai. On the order of uniprimitive permutation groups. Annals of Mathematics, 113(3):553-568, 1981.

[4] Z. Beerliova, F. Eberhard, T. Erlebach, A. Hall, M. Hoffmann, M. Mihalák, and L. S. Ram. Network discovery and verification. IEEE Journal on Selected Areas in Communications, 24(12):2168-2181, 2006.

[5] J. Cáceres, M. C. Hernando, M. Mora, I. M. Pelayo, M. L. Puertas, C. Seara, and D. R. Wood. On the metric dimension of cartesian products of graphs. SIAM Journal on Discrete Mathematics, 21(2):423-441, 2007.

[6] G. Chartrand, L. Eroh, M. A. Johnson, and O. R. Oellermann. Resolvability in graphs and the metric dimension of a graph. Discrete Applied Mathematics, 105(1-3):99-113, 2000.

[7] G. Chartrand and P. Zhang. The theory and applications of resolvability in graphs: A survey. Congressus Numerantium, 160:47-68, 2003.

[8] V. Chvátal. Mastermind. Combinatorica, 3(3):325-329, 1983.

[9] J. Díaz, O. Pottonen, M. J. Serna, and E. J. van Leeuwen. Complexity of metric dimension on planar graphs. Journal of Computer and System Sciences 83(1):132-158, 2017.

[10] L. Epstein, A. Levin, and G. J. Woeginger. The (weighted) metric dimension of graphs: Hard and easy cases. Algorithmica, 72(4):1130-1171, 2015.

[11] F. Harary and R. Melter. The metric dimension of a graph. Ars Combinatoria, 2:191-195, 1976.

[12] M. Hauptmann, R. Schmied, and C. Viehmann. Approximation complexity of metric dimension problem. Journal of Discrete Algorithms, 14:214-222, 2012.

[13] V. Jude Annie Cynthia. Metric dimension of certain mesh derived graphs. Journal of Mathematical and Computational Science Vol.5 (1), 71-77, 2014 
[14] S. Khuller, B. Raghavachari, and A. Rosenfeld. Landmarks in graphs. Discrete Applied Mathematics, 70(3):217-229, 1996.

[15] R. A. Melter and I. Tomescu. Metric bases in digital geometry. Computer Vision, Graphics, and Image Processing, 25:113-121, 1984.

[16] A. Sebö and E. Tannier. On metric generators of graphs. Mathematics of Operations Research, 29(2):383-393, 2004.

[17] B. Shanmukha, B. Sooryanarayana, and K. S. Harinath. Metric dimension of wheels. Far East Journal of Applied Mathematics, 8(3):217-229, 2002.

[18] P. J. Slater. Leaves of trees. Congressus Numerantium, 14:549-559, 1975.

Received 1st February 2018 